\title{
Diagnostic Accuracy of CRP \& WCC in Abdomino-Pelvic Diseases of Non-Traumatic Acute Abdomen by Taking CT Findings as Gold Standard
}

\author{
Chauhan MN, Rehman S, Riaz M, J onker L, Ali D, \\ Zaman $S$ and Canelo $\mathbf{R}^{*}$ \\ Department of General \& Hepatobiliary Surgery, \\ Cumberland Infirmary Carlisle, UK \\ *Corresponding author: Ruben Canelo FRCS, \\ Department of Hepatobiliary Surgery, Cumberland \\ Infirmary Carlisle, Infirmary Road, Carlisle CA2 7HY, UK
}

Received: March 01, 2018; Accepted: March 21, 2018; Published: March 28, 2018

\begin{abstract}
Acute abdomen is a common presentation to many emergency departments worldwide. It is important to differentiate self- limiting causes and serious surgical causes. Inflammatory markers such as WCC and CRP currently being used to determine the cause, assess severity and guide further investigation for acute abdomen. The aim of this study was to determine diagnostic accuracy of $C$ - reactive protein and White Cell Count in abdomino-pelvic diseases of non-traumatic acute abdomen by taking $\mathrm{CT}$ scan findings as gold standard. We found that CRP levels of above 100 were highly sensitive (94\%) and specific $(92 \%)$ for an acute surgical abdomen. WCC was not as accurate at determining an acute surgical abdomen due to reasons such as neutropenic sepsis.
\end{abstract}

\section{Introduction}

Abdominal pain accounts for $5-10 \%$ of presentation to the $\mathrm{A} \& \mathrm{E}$ department [1]. The "acute abdomen" is defined as "sudden severe abdominal pain of unknown origin" and is a very common cause for presentation to emergency department worldwide [1-5]. Diagnosis is dependent upon clinical assessment and investigations [3]. Clinical assessment involves a thorough history and clinical examination and is subject to variability based on clinical experience of examining physician [2]. Clinical examination has only been found to be accurate $47-76 \%$ of the time with only $50 \%$ of junior doctors and doctors working in community reaching a true diagnosis from the above alone [2]. Delay in diagnosis of acute abdomen affects instigation of treatment resulting in increased morbidity and mortality [5-7]. Serological markers of inflammation including $\mathrm{C}$ - reactive protein (CRP) and White Cell Count (WCC) are often used by clinicians as base line investigation to narrow down the differential diagnosis and guide further investigations including imaging.

The role of raise CRP and WCC have been extensively investigated with regards to their role in diagnosing acute appendicitis [3-5]. For example, studies have shown CRP sensitivities in acute appendicitis ranging from $40-94 \%$ and specificities ranging from $38-87 \%$ [3-5]. However, little have been reported regarding the role of these two inflammatory markers in assessing patients presenting with acute abdomen in non-trauma abdomino-pelvic diseases. Sengupta et al. suggest that patients experiencing lower abdominal pain, with normal WCC and CRP, can be sent home [6]. CRP unlike WCC is an 'add on' blood marker in many trusts, not used routinely in $\mathrm{A} \& \mathrm{E}$ blood profiles.

Acute abdomen is a term frequently used to describe the acute abdominal pain in a subgroup of patients who are seriously ill and have abdominal tenderness and rigidity. Acute abdominal pain or disease may be caused by a myriad of diagnoses, including acute appendicitis, diverticulitis, cholecystitis and perforated bowel [10]. Computed Tomography (CT) of the abdomen and pelvis is considered the most appropriate examination for patient's abdomino-pelvic diseases $[11,12]$. Non-enhanced CT, US, and conventional radiography are considered less appropriate initial imaging examinations for these patients [10]. But Ionizing radiation exposure at CT is associated with the risk of radiation induced cancer. This is a drawback of CT, especially as CT is increasingly being used in the diagnostic work-up of young patients [13].

Physicians in the emergency department often base their decisions for consultation of the surgeon for a laparotomy on clinical presentation combined with biochemical abnormalities. Examples of biochemical parameters include raised C-reactive protein (CRP) [14]. C-reactive protein (CRP) is a non-specific inflammatory marker that is used routinely in many hospitals as an aid in the diagnosis of patients with an acute abdomen [15]. One recent study conclude that biomarkers lactate and CRP in patients with acute abdominal pain should only be used in adjunct to the history and clinical findings, as they are not specific and can be misleading in establishing a diagnosis. In addition, relying on these biomarkers may contribute to more diagnostic examinations and/or unnecessary invasive interventions (for example laparotomy) [16].

Amore recent study reported the sensitivity of CRP (> $5 \mathrm{mg} / \mathrm{l})$ $81.7 \%$ and the specificity $48.5 \%$ in patients with abdomino-pelvic diseases, they reported CRP at $48 \mathrm{~h}$ after hospital admission showed a good prognostic accuracy and can be used as an alternative to CT scan [17].

The rationale of this study is to determine diagnostic accuracy of $\mathrm{C}$ - reactive protein in abdomino-pelvic diseases of non-traumatic acute abdomen taking CT findings as gold standard. There are very limited studies available in the literature with the role of C-reactive in abdomino-pelvic diseases. This study will help us to provide a guide for an early diagnosis, so that patient may have an early diagnosis and successful treatment.

This study aimed to evaluate diagnostic accuracy, sensitivity and specificity of raised CRP and WCC both individually as well
Austin J Surg - Volume 5 Issue 5 - 2018

ISSN : 2381-9030 | www.austinpublishing group.com

Canelo et al. () All rights are reserved
Citation: Chauhan MN, Rehman S, Riaz M, Jonker L, Ali D, Zaman S, et al. Diagnostic Accuracy of CRP \& WCC in Abdomino-Pelvic Diseases of Non-Traumatic Acute Abdomen by Taking CT Findings as Gold Standard. Austin J Surg. 2018; 5(5): 1142. 


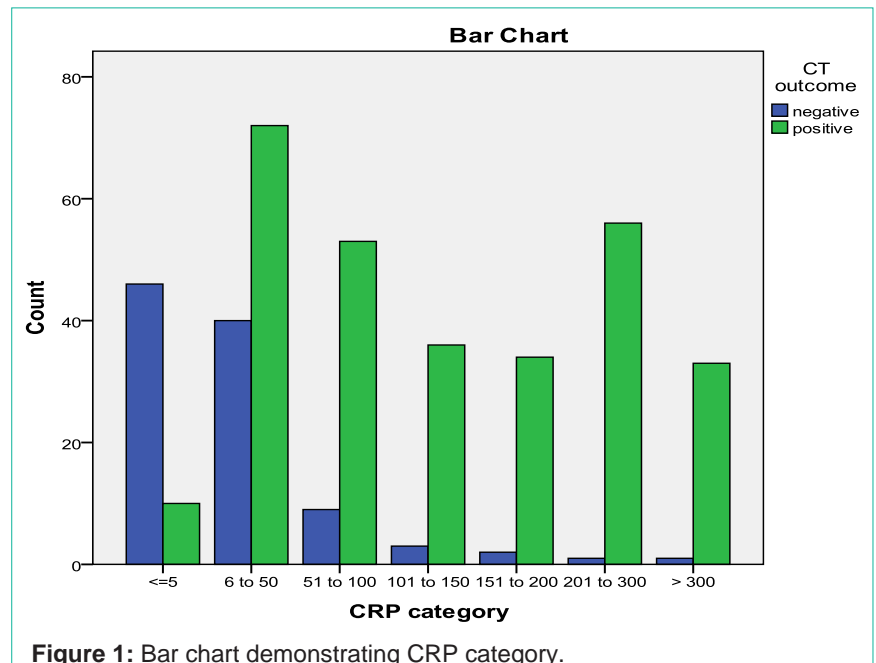

Figure 1: Bar chart demonstrating CRP category.

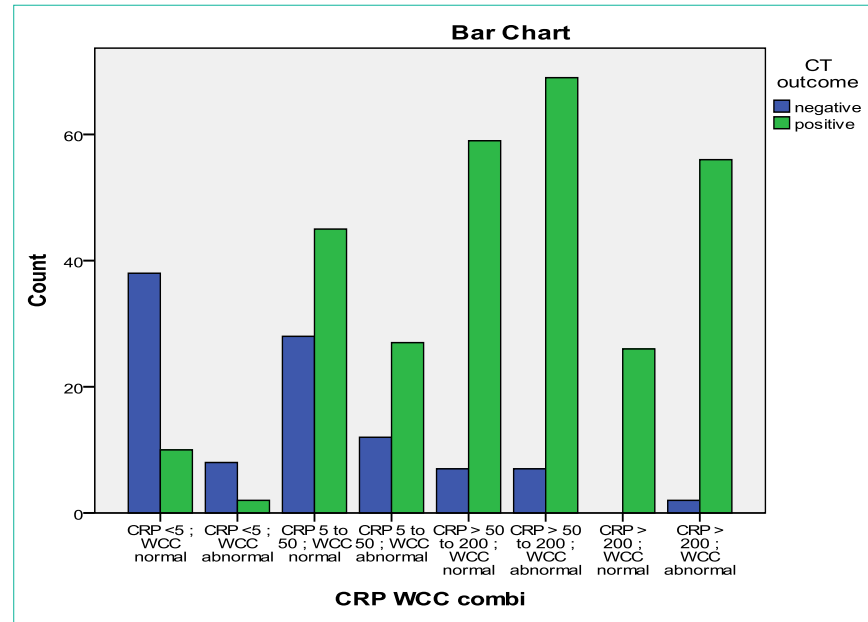

Figue 2: Bar chart demonstrating CRP Values.

as collectively in patients presenting with acute abdomen of nontraumatic origin. Comparison was made with CT scan abdomen findings.

\section{Method}

We retrospectively evaluated patients admitted with acute abdominal pain to the surgical department between January 2014 to June 2014.Data was entered on a structured proforma. Eligible patients were assessed and an initial diagnosis was made depending on a combination of history, clinical examination, laboratory tests and initial plain radiography. The final diagnosis was confirmed with CT scan. In total 396 patients were included with all basic demographics, such as name, age and sex being noted. CRP and WCC values within 24 hours of admission were recorded and its diagnostic value was then compared to CT scan results. CRP $>5 \mathrm{mg} / \mathrm{l}$ and WCC $>11$ or $<4$ were set as being abnormal as per standard recommendation and literature reports.

The laboratory method used to measure CRP in serum was polyethylene glycol (PEG) enhanced immune-turbid metric assay. The CRP level within 24 hours of admission was recorded and its diagnostic value compared to the final diagnosis of all patients.
Data entry and analysis was performed using SPSS 20. We used chi-square test to compare continuous variable and Mann-Whitney $\mathrm{U}$ test for categorical variables.

\section{Results}

A total of 396 patients fulfilled the inclusion criteria; 237 were females and 159 were males with a mean age of 59 years. $74 \%$ patient had positive CT scan results i.e. results in actual diagnosis rather than non-specific abdominal pain.

One hundred and fifty four cases had abnormal WCC $(>11$ or $<4)$ and a positive CT scan abdomen findings. The specificity and sensitivity of WCC was therefore calculated as $72 \%$ and $52 \%$ respectively. The Pearson Chi-Square: p-value $<0.001$ is highly significant; i.e. there is a trend that +ve CT is associated with abnormal WCC.

Table 1 highlights the specificity and sensitivity of CRP values only. Both the specificity and sensitivity increase significantly when CRP levels rose above 51. However, CRP levels between 6-50 were only $53 \%$ sensitive and $64 \%$ specific.

Moreover, Figure 1 demonstrates the increasing positive CT outcome with higher CRP values. At $\mathrm{CRP}<5$ there are more negative $\mathrm{CT}$ scan results shown in blue than positive results, shown in green. A rise in CRP level results in positive CT findings.

In addition, Table 2 and Figure 2 above show the specificity and sensitivity of CRP combined with WCC values. As proved, CRP values were more specific and sensitive than WCC in the acute abdomen. This is highlighted by when the $\mathrm{CT}$ scan result was positive in 26/26 cases where the CRP levels were $>200$ but with normal WCC.

Furthermore, Table 3 below compares sensitivities and specificities of CRP along with WCC and CRP alone. There is not a significant difference in results when CRP levels are between 5-50 with abnormal WCC. But as mentioned previously, as CRP levels raise its significance in diagnosing an acute abdomen raises more than a rise in WCC value. Various pathologies were diagnosed during the study following the CT scan report. Table 3 and Figures 3 \& 4 below highlight the distribution of pathology.

\section{Discussion}

The acute abdomen remains a diagnostic challenge for many surgeons. Raised CRP and WCC alone cannot indicate whether the cause of abdominal pain may require operative intervention or if it is self-limiting as found recently by Salam et al. [7]. That is the critism that some studies have issued against using CRP as a diagnostic tool in the surgical abdomen. Andersson et al. in their study reported that

Table 1: Values of CRP and Specificity and Sensitivity.

\begin{tabular}{|c|c|c|}
\hline Category (CRP only) & Specificity & Sensitivity \\
\hline$=<5$ & n/a & n/a \\
\hline 6 to 50 & $64 \%(72 / 112)$ & $53 \%(46 / 86)$ \\
\hline 51 to 100 & $85 \%(53 / 62)$ & $84 \%(46 / 55)$ \\
\hline 101 to 150 & $92 \%(36 / 39)$ & $94 \%(46 / 49)$ \\
\hline 151 to 200 & $94 \%(34 / 36)$ & $96 \%(46 / 48)$ \\
\hline 200 to 300 & $98 \%(56 / 57)$ & $98 \%(46 / 47)$ \\
\hline$>300$ & $97 \%(33 / 34)$ & $98 \%(46 / 47)$ \\
\hline
\end{tabular}


Table 2: CRP levels and specificity and sensitivity.

\begin{tabular}{|c|c|c|}
\hline $\begin{array}{c}\text { Category * } \\
\text { CRP }<5 \text { and WCC normal (reference } \\
\text { category) }\end{array}$ & n/a [reference] & n/a [reference] \\
\hline CRP $<5$ and WCC abnormal & $83 \%(38 / 46)$ & $20 \%(2 / 10)$ \\
\hline CRP $>5$ to 50 and WCC normal & $58 \%(38 / 66)$ & $62 \%(45 / 73)$ \\
\hline CRP $>5$ to 50 and WCC abnormal & $76 \%(38 / 50)$ & $69 \%(27 / 39)$ \\
\hline CRP $>50$ to 200 and WCC normal & $84 \%(38 / 45)$ & $89 \%(59 / 66)$ \\
\hline CRP $>50$ to 200 and WCC abnormal & $84 \%(38 / 45)$ & $91 \%(69 / 76)$ \\
\hline CRP $>200$ and WCC normal & $100 \%(38 / 38)$ & $100 \%(26 / 26)$ \\
\hline CRP $>200$ and WCC abnormal & $95 \%(38 / 40)$ & $97 \%(56 / 58)$ \\
\hline
\end{tabular}

Table 3: Frequency of Diagnosis on CT findings.

\begin{tabular}{|c|c|}
\hline Diagnosis & Frequency \\
\hline Diverticulitis & 36 \\
\hline Small bowel obstruction & 36 \\
\hline Acute Cholecystitis & 28 \\
\hline Colitis & 24 \\
\hline Acute pancreatitis & 21 \\
\hline Dilated CBD +/- Cholangitis & 16 \\
\hline Acute Appendicitis & 20 \\
\hline Tubo ovarian /Adnexal mass & 12 \\
\hline Colonic malignancy & 11 \\
\hline Small bowel inflammation (Cohn's ileitis) & 10 \\
\hline Intra abdominal collection & 10 \\
\hline Negqtive CT & 102 \\
\hline
\end{tabular}

CRP alone was not useful in differentiating a self-limiting condition like NSAP (non-specific abdominal pain) from other important surgical causes of acute abdomen [8] whilst surprisingly high levels of CRP was found in patients who would have normally been treated as having NSAP [8]. NSAP is short-lived condition of unknown cause that settles spontaneously with no long-term consequences in majority of the patients at long-term follow-up. High levels of CRP in patients with NSAP may indicate that NSAP is actually an inflammatory condition. However, this adds little or nothing to the fact that such high levels of CRP cannot differentiate between this selflimiting condition and more potentially serious surgical diagnosis.

In our study we are not using CRP or WCC as a diagnostic tool alone. We are using them as an adjunct to reach final diagnosis which is confirmed by the CT scan abdomen. When used in this manner, we found that CRP can be very useful with high sensitivities and specificities as mentioned

From the results we concluded 2 main points;

1. A CRP of 6-50 with abnormal WCC has a better diagnostic value then CRP alone.

CRP 6-50 + abnormal WCC $=$ Specificity 76\%, Sensitivity $69 \%$.

CRP 6-50 + normal WCC = specificity 58\%, sensitivity $62 \%$.

CRP 6-50 (alone) = specificity $64 \%$, sensitivity $53 \%$.

Abnormal WCC alone $=$ specificity $72 \%$, sensitivity $52 \%$.

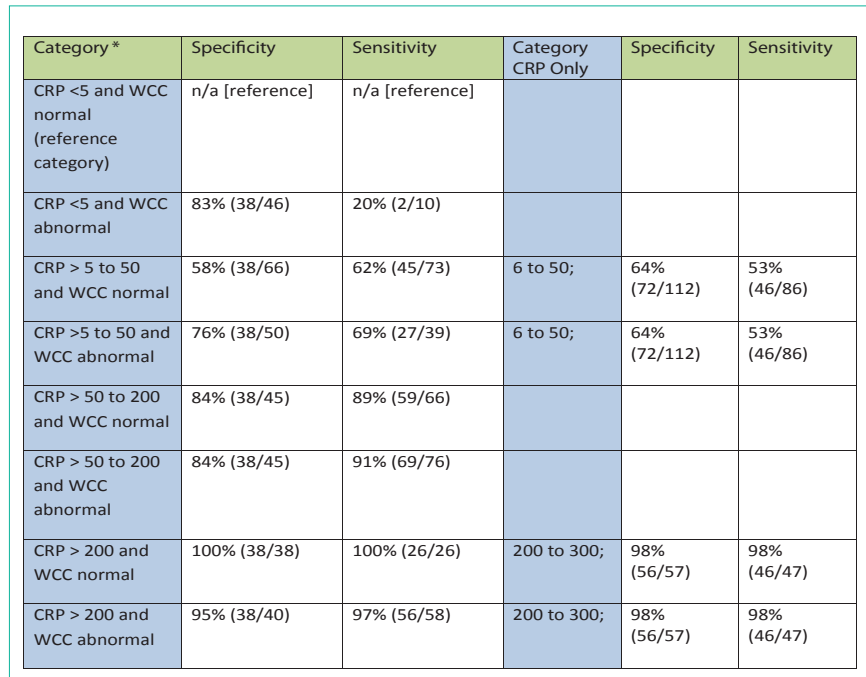

Figure 3: CRP sensitivity and specificity.

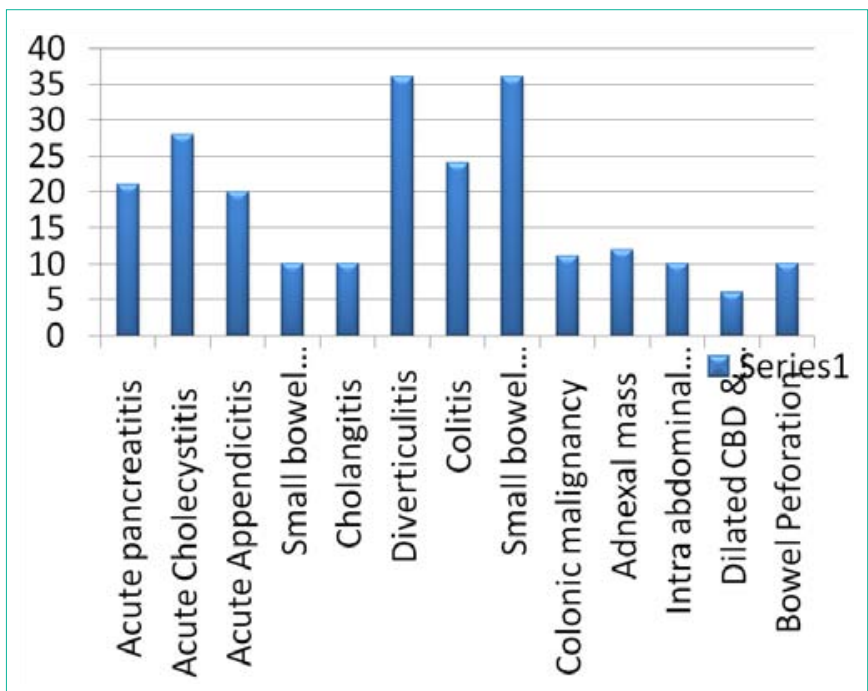

Figure 4: CRP values and CT Findings.

2. CRP alone of 100 or more has better specificity and sensitivity than other two combinations

CRP $51-100$ specificity $=85 \%$, sensitivity $=84 \%$

CRP $101-150$ specificity $=92 \%$, sensitivity $=94 \%$

CRP $>200=$ specificity, sensitivity of $98 \%$ each.

This shows that CRP levels above 50 have an increased sensitivity and specificity. The possible reason for CRP being superior to WCC in diagnosing a surgical abdomen is that patients can become neutropenic causing WCC levels to decrease, but CRP remains elevated.

Apart from WCC and CRP, lactate levels can also indicate a surgical abdomen. An increase of lactate levels indicates an anaerobicglycogenesis' and therefore it is a parameter for inadequate perfusion, oxygenation and an estimate of tissue oxygen deficiency. Increased plasma lactate concentrations were observed in patients with mesenteric ischemia with a sensitivity of $100 \%$ and a specificity 
of $42 \%$ [9]. Other studies have shown raised lactate levels can also be due to other surgical conditions such as bowel obstruction and peritonitis. A further study in the comparison of lactate and CRP in the surgical abdomen may prove of benefit. CRP values above 100 are significant and patient with such values with a history of acute abdominal pain should undergo a CT scan to further evaluate the cause, which may be surgical or could well be self-limiting.

\section{References}

1. Emergency education. 2017.

2. Ng CS, Watson CJE, Palmer CR, See TC, Beharry NA, Housdon BA, et al. Evaluation of early abdominopelvic computed tomography in patients with acute abdominal pain of unknown cause: prospective randomized study. BMJ. 2002; 325: 1387.

3. Al-Saigh $\mathrm{AH}$. C-reactive protein in the differential diagnosis of the acute abdomen, especially acute appendicitis. J R CollSurgEdinb. 1992; 37: 238240 .

4. Gurleyik E, Gurleyik G, Unalmiser S. Accuracy of serum C-reactive protein measurements in diagnosis of acute appendicitis compared with surgeon's clinical impression. Dis Colon Rectum. 1995; 38: 1270-1274.

5. Yang HR, Wang YC, Chung PK, Chen WK, Jeng LB, Chen RJ. Role of leukocyte count, neutrophil percentage, and C-reactive protein in the diagnosis of acute appendicitis in the elderly. Am Surg. 2005; 71: 344-347.

6. Sengupta A, Bax G, Paterson-Brown S. White cell count and C-reactive protein measurement in patients with possible appendicitis. Ann R Coll Surg Engl. 2009; 91: 113-115.

7. Salem TA, Molloy RG, O'Dwyer PJ. Prospective study on the role of C-reactive protein (CRP) in patients with an acute abdomen. Ann R Coll Surg Engl. 2007; 89: 233-237.

8. Andersson RE. Meta-analysis of the clinical and laboratory diagnosis of appendicitis. Br J Surg. 2004; 91: 28-37.
9. Lange $\mathrm{H}$, Toivola $\mathrm{A}$. Warning signals in acute abdominal disorders. Lactate is the best marker of mesenteric ischemia. Lakartidningen. 1997; 94: 18931896.

10. Stoker J, van Randen A, Laméris W, Boermeester MA. Imaging Patients with Acute Abdominal Pain ${ }^{1}$. Radiology. 2009; 253: 31-46.

11. Van Randen A, Laméris W, Nio CY, Spijkerboer AM, Meier MA, Nolthenius $\mathrm{CT}$, et al. Inter-observer agreement for abdominal CT in unselected patients with acute abdominal pain. Eur Radiol. 2009; 19: 1394-1407.

12. Matz S, Connell M, Sinha M, Goettl CS, Patel PC, Drachman D. Clinical Outcomes of Pediatric Patients With Acute Abdominal Pain and Incidental Findings of Free Intraperitoneal Fluid on Diagnostic Imaging. J Ultrasound Med. 2013; 32: 1547-1553.

13. Stoker J. Magnetic resonance imaging and the acute abdomen. Br J Surg. 2008; 95: 1193-1194.

14. Ravishankaran P, Shah AM, Bhat R. Correlation of interleukin-6, serum lactate, and C-reactive protein to inflammation, complication, and outcome during the surgical course of patients with acute abdomen. J Interferon Cytokine Res. 2011; 31: 685-690.

15. Salem T, Molloy R, O'dwyer P. Prospective study on the role of C-reactive protein (CRP) in patients with an acute abdomen. Ann $\mathrm{R}$ Coll Surg Engl. 2007; 89: 233-237.

16. Meyer ZC, Schreinemakers JM, van der Laan L. The value of C-reactive protein and lactate in the acute abdomen in the emergency department. World Journal of Emergency Surgery. 2012; 7: 22.

17. Coyle JP, Brennan CR, Parfrey SF, O'Connor OJ, Mc Laughlin PD, Mc Williams SR, et al. Is serum $\mathrm{C}$-reactive protein a reliable predictor of abdomino-pelvic CT findings in the clinical setting of the non-traumatic acute abdomen?. Emerg radiol. 2012; 19: 455-462.
Austin J Surg - Volume 5 Issue 5 - 2018

ISSN : 2381-9030 | www.austinpublishinggroup.com

Canelo et al. (C) All rights are reserved
Citation: Chauhan MN, Rehman S, Riaz M, Jonker L, Ali D, Zaman S, et al. Diagnostic Accuracy of CRP \& WCC in Abdomino-Pelvic Diseases of Non-Traumatic Acute Abdomen by Taking CT Findings as Gold Standard. Austin J Surg. 2018; 5(5): 1142. 